\title{
The formation of interstellar molecular lines in a turbulent velocity field with finite correlation length
}

\section{Physical parameters of Bok Gobules}

\author{
M. Hegmann ${ }^{1}$, C. Hengel ${ }^{2}$, M. Röllig ${ }^{3}$, and W. H. Kegel ${ }^{1}$ \\ 1 Zentrum für Astronomie und Astrophysik, Technische Universität Berlin, Hardenberg 36, 10623 Berlin, Germany \\ e-mail: hegmann@astro.physik.tu-berlin.de \\ 2 Institut für Theoretische Physik, Universität Frankfurt, Robert-Mayer-Strasse 8-10, 60054 Frankfurt, Germany \\ 3 I. Physikalisches Institut der Universität zu Köln, Zülpicher Straße 77, 50937 Köln, Germany
}

Received 23 August 2004 / Accepted 26 August 2005

ABSTRACT

We present observational line data for five Bok Globules. Observations were made in the $J=2 \rightarrow 1$ and $J=3 \rightarrow 2$ rotational transitions of $\mathrm{CO},{ }^{13} \mathrm{CO}$ and $\mathrm{C}^{18} \mathrm{O}$ at the Heinrich-Hertz-Telescope in Arizona. Using the stochastic radiative transfer model (SRTM) based on the generalized radiative transfer equation as described in the previous papers of this series, we were able to fit the observed CO lines for four of the five Bok globules. The derived parameters such as the hydrogen density, the cloud mass and temperature in part differ significantly from values obtained from a standard LVG analysis or a microturbulent approach. The best fits are obtained for models characterized by a ratio of the mean free path of photons to the correlation length of the turbulent velocity field of the order of unity. This is what characterizes the mesoturbulent regime that cannot be adressed by standard evaluation methods like LVG or microturbulent approximations.

Key words. radiative transfer - turbulence - ISM: clouds - ISM: lines and bands

\section{Introduction}

The quantitative interpretation of interstellar molecular lines is the most important tool to derive physical parameters of molecular clouds. To calculate theoretical line profiles which then may be compared with observations, one has at first to specify a reasonable physical model which on one side is simple enough to be numerically tractable, and on the other side contains enough physics that a comparison is meaningful. Of particular importance is the line broadening mechanism. Due to the low density in molecular clouds, Doppler broadening is the by far the most important broadening mechanism. In most cases the observed line width is considerably larger than the thermal width. This implies that large non-thermal velocities are present in the clouds. These may be either systematic (e.g. in collapsing clouds) or stochastic or both. Most calculations are done assuming one or other extreme, i.e. either by assuming the line broadening to be solely due to systematic motion (see e.g. Goldreich \& Kwan 1974) or to turbulence (see e.g. Leung \& Liszt 1976). In the first case it is common to use an LVG-code, which simplifies the numerical calculations considerably, but assumes a very specific velocity field and a homogeneous density in its most widely used form. In its more general form, as originally formulated by Sobolev (1967), the method allows for an escape probability depending on position and direction and by this allows for more general physical models. Since the actual structure of molecular clouds is extremely complex and in order to limit the numerical effort, it is common practice in the second case to characterize the velocity field by its statistical properties only. Most investigations considering turbulent line broadening are based on the microturbulent approximation in which the velocity field is characterized by one parameter only, the mean turbulent velocity $\sigma$. In the previous papers of this series (Albrecht \& Kegel 1987, Paper I; Kegel et al. 1993, Paper II; Piehler \& Kegel 1995, Paper III) we studied line formation in a turbulent medium accounting in lowest order for the influence of a finite correlation length. In this mesoturbulent approximation (the Stochastic Radiative Transfer Model, SRTM) the velocity field is characterized by two parameters, the average turbulent velocity $\sigma$ and the correlation length $\ell$. We showed that taking into account correlation effects has a significant influence on line intensities, line ratios and line shapes. These effects are as important as NLTE-effects.

In the present paper, we present an analysis of millimeter line data taken for a set of Bok Globules. Observations were made in 1999-2001 at the Heinrich-Hertz-Telescope in Arizona in the rotational transitions of $\mathrm{CO},{ }^{13} \mathrm{CO}$ and $\mathrm{C}^{18} \mathrm{O}$. We analyze the data by means of our stochastic radiative transfer 
model (SRTM) as specified in Paper III. Although this stochastic approximation made in the frame of the SRTM still implies major simplifications, the treatment of the radiative transfer in the SRTM is much more sophisticated than in the other approximations mentioned above. In this sense, the SRTM can be considered as a complementary approach, emphasizing mainly radiative transfer effects. This paper has two principal objectives: We examine to what extent the SRTM in its present form is suitable for the analysis of millimeter and submillimeter line data. Second, we compare the results determined by means of the SRTM to the parameters derived from an LVG-analysis or a microturbulent approximation for the velocity field (Turner 1994).

\section{The stochastic radiative transfer model}

\subsection{Stochastic description of the turbulent velocity field}

In this section, we give a brief outline of the stochastic radiative transfer model. For an in-depth discussion we refer to Paper II and references therein. We consider the transport of radiation in a turbulent medium under the assumption that the turbulent velocity field can be described in terms of its stochastic properties. This means that the functional form of the multipoint probability distributions has to be given. Following Gail et al. (1974), we assume a Markovian structure for the velocity field along each line of sight. In this approximation only two-point correlations are taken into account, and the stochastic velocity field is completely defined by specifying the one-point distribution of the velocity and the corresponding two-point correlation function. We consider the one-point distribution of $v$ (the turbulent velocity component along the line of sight) to be Gaussian

$W_{1}(v)=\frac{1}{\sqrt{2 \pi} \sigma} \exp \left(-\frac{v^{2}}{2 \sigma^{2}}\right)$

and the two-point correlation function to be exponential

$f(\Delta s)=\frac{\langle v(s) v(s+\Delta s)\rangle}{\sigma^{2}}=\exp \left(-\frac{|\Delta s|}{\ell}\right)$,

where $\sigma^{2}$ and $\ell$ are the mean quadratic turbulent velocity and the correlation length of the velocity field, respectively.

\subsection{The generalized radiative transfer equation}

Due to the stochastic nature of the underlying velocity field the intensity becomes a stochastic variable, too. It can be shown that Eqs. (1) and (2) together with the ordinary radiative transfer equation

$\frac{\mathrm{d} I_{v}}{\mathrm{~d} s}=-\kappa_{v}\left(I_{v}-S_{v}\right)$

lead to a Fokker-Planck equation for the conditional intensity $q_{v}(v)$ (Gail et al. 1974):

$\frac{\partial q_{v}}{\partial s}=\frac{1}{\ell}\left(-v \frac{\partial q_{v}}{\partial v}+\sigma^{2} \frac{\partial^{2} q_{v}}{\partial v^{2}}\right)-\kappa_{v}\left(q_{v}-S_{v}\right)$.
The actual expectation value of the intensity can be calculated from $q_{v}(v)$ by

$\left\langle I_{v}\right\rangle=\int q_{v}(v) W_{1}(v) \mathrm{d} v$.

The source function $S_{v}(v, s)$ and the absorption coefficient $\kappa_{v}(v, s)$ are given in the usual way by the occupation numbers $n_{i}$ and $n_{j}$ corresponding to the transition considered. This means that in the case of NLTE, the generalized transfer equation has to be solved consistently with the set of rate equations (for a detailed derivation see Gail et al. 1975):

$$
\begin{aligned}
\sum_{j \neq i}\left(n_{j}(v)\right. & \left.\left(A_{i j}+C_{i j}\right)-n_{i}(v)\left(A_{j i}+C_{j i}\right)\right) \\
& +\sum_{j \neq i}\left(\frac{4 \pi}{c}\left\langle\widetilde{J}_{i j}(v)\right\rangle\left(n_{j}(v) B_{i j}-n_{i}(v) B_{j i}\right)\right)=0 .
\end{aligned}
$$

$A_{i j}$ and $B_{i j}$ are the Einstein coefficients for spontaneous and induced transitions, while $C_{i j}$ denotes the probabilities for collisional transitions. For the solid angle average of the intensity, we use the following approximative term introduced by Albrecht and Kegel in Paper I:

$\left\langle\widetilde{J}_{i j}(v)\right\rangle \approx \frac{1}{4 \pi} \iint\left[q_{v}(v)\right]_{i j} \Phi(v, v) \mathrm{d} v \mathrm{~d} \Omega$,

where $\Phi(v, v)$ is the normalized profile of the local absorption coefficient under the assumption of complete redistribution over this local profile.

\section{Cloud model}

As the underlying cloud model, we use the model of an isothermal sphere described by Piehler \& Kegel (1995).

The basic idea is to describe the cloud as a gas sphere in hydrostatic equilibrium, being stabilized against gravitational collapse by thermal and turbulent pressure. The fundamental equations determining the cloud structure are the hydrostatic equation, the Poisson-equation in its integral representation and an equation of state:

$\frac{\mathrm{d} p}{\mathrm{~d} r}=-\frac{G M(r) \rho(r)}{r^{2}}$

$\frac{\mathrm{d} M(r)}{\mathrm{d} r}=4 \pi r^{2} \rho(r)$,

$p(r)=\left(\frac{k_{\mathrm{B}} T}{m}+\sigma^{2}\right) \rho(r)$,

using the following abbreviations:

\section{$r$ distance from cloud center}

$M(r)$ mass internal to the radius $r$

$\rho(r)$ local mass density

$p(r)$ local gas presure

$T \quad$ kinetic gas temperature

$\sigma \quad$ mean turbulent velocity

$m$ mean molecular mass

$G \quad$ gravitational constant.

Since an isothermal cloud can only be stable at non-zero external pressure, we define the cloud radius $R_{\mathrm{H}_{2}}$ to be the radius for which the local cloud pressure equals the pressure 
Table 1. The selected sources and their coordinates: 1950.0 right ascension and declination and the measured radial velocity of the cloud.

\begin{tabular}{lllc}
\hline \hline Source & $\alpha_{1950}$ & $\delta_{1950}$ & $v_{\text {LSR }}\left[\mathrm{km} \mathrm{s}^{-1}\right]$ \\
\hline CB 24 & $4^{\mathrm{h}} 54^{\mathrm{m}} 33^{\mathrm{s}}$ & $52^{\circ} 11^{\prime} 07^{\prime \prime}$ & 4.6 \\
CB 25 & $4^{\mathrm{h}} 55^{\mathrm{m}} 08^{\mathrm{s}}$ & $51^{\circ} 58^{\prime} 52^{\prime \prime}$ & 5.2 \\
CB 202 & $19^{\mathrm{h}} 40^{\mathrm{m}} 18^{\mathrm{s}}$ & $18^{\circ} 45^{\prime} 00^{\prime \prime}$ & 18.1 \\
CB 213 & $19^{\mathrm{h}} 59^{\mathrm{m}} 24^{\mathrm{s}}$ & $24^{\circ} 34^{\prime} 24^{\prime \prime}$ & 7.6 \\
CB 231 & $21^{\mathrm{h}} 32^{\mathrm{m}} 18 \mathrm{~s}$ & $54^{\circ} 28^{\prime} 06^{\prime \prime}$ & 6.5 \\
\hline
\end{tabular}

of the mean interstellar medium $p_{\text {ISM }}$. We adopt a value of $p_{\text {ISM }}=3 \times 10^{-13} \mathrm{dyn}^{-2}$ as given by Blitz (1987). In the hydrostatic equilibrium model, we account for the molecular hydrogen component only. For the CO-radiative transfer problem however, we have to define a smaller cloud radius $R_{\mathrm{CO}}$, because in the outer part of the molecular cloud CO is dissociated by the ambient interstellar FUV radiation field. $R_{\mathrm{CO}}$ is given by $R_{\mathrm{H}_{2}}$ diminished by the depth for which the visual extinction becomes $A_{\mathrm{V}}=1.7$ (cf. van des Hoeck \& Black 1988), i.e. $N_{\mathrm{H}_{2}}=10^{21} \mathrm{~cm}^{-2}$ measured from the cloud edge. Throughout this smaller sphere with radius $R_{\mathrm{CO}}$ we assume a fixed $\mathrm{CO} / \mathrm{H}_{2}$ ratio of $8 \times 10^{-5}$. As long as the abundances remain fixed, the complete model consisting of the cloud model and the SRTM contains four free parameters, the central number density of molecular hydrogen $n_{0}$, the cloud temperature $T$, the mean turbulent velocity $\sigma$ and the correlation length of the velocity field $\ell$.

\section{Observations}

We scanned the catalogue of Clemens \& Barvanis (1989) for starless and essentially spherical Bok Globules, because these simply structured and isolated objects appear to fulfill our model assumptions best. The candidates we finally selected for investigation are listed in Table 1. The distances of the globules are not known in general. Clemens and Barvanis argued them to be less than $1 \mathrm{kpc}$, and probably $\approx 600 \mathrm{pc}$ on average. For CB 24, Peterson and Clemens (1998) were able to determine a maximum distance of $360 \mathrm{pc}$.

Observations were made in 1999, 2000 and 2001 at the Heinrich-Hertz-Telescope (HHT) of the SMTO in Arizona. We observed in the $\mathrm{CO}(2-1)(230.54 \mathrm{GHz}), \mathrm{CO}(3-2)$ (345.80 GHz), ${ }^{13} \mathrm{CO}(2-1) \quad(220.40 \mathrm{GHz}),{ }^{13} \mathrm{CO}(3-2)$ $(330.59 \mathrm{GHz})$ and $\mathrm{C}^{18} \mathrm{O}(2-1)(219.56 \mathrm{GHz})$ transitions, using the SIS 230 and SIS 345 receivers of the HHT. The measurements were made in position switching mode. The planned CO (4-3) observation could not be accomplished in all three observing runs due to bad weather conditions. We improved the quality of the calibration by using secondary calibration sources.

We used the filterbank spectrometer (FBS) with a channel width of $0.0625 \mathrm{MHz}$ corresponding to $\approx 0.08 \mathrm{~km} \mathrm{~s}^{-1}$ for the SIS 230 and $\approx 0.05 \mathrm{~km} \mathrm{~s}^{-1}$ for the SIS 345 receiver. Receiver temperatures were approximately $130 \mathrm{~K}$ at $230 \mathrm{GHz}$ and $200 \mathrm{~K}$ at $345 \mathrm{GHz}$; system temperatures were $300-500 \mathrm{~K}$ and $500-1000 \mathrm{~K}$ on a $T_{\mathrm{A}}^{\star}$ scale, respectively.
Table 2. Calibration factors for the conversion of $T_{\mathrm{A}}^{\star}$ into approximate brightness temperatures $T_{\mathrm{B}}$ (D. Muders, priv. comm.).

\begin{tabular}{ccccc}
\hline \hline$v[\mathrm{GHz}]$ & $\lambda[\mathrm{mm}]$ & $\eta_{\mathrm{mb}}$ & $\eta_{\mathrm{f}}$ & $\eta_{\mathrm{f}} / \eta_{\mathrm{mb}}$ \\
\hline 230 & 1.3 & 0.78 & 0.95 & 1.22 \\
345 & 0.9 & 0.47 & 0.90 & 1.91 \\
460 & 0.65 & 0.48 & 0.78 & 1.63 \\
\hline
\end{tabular}

The typical diameter of the selected sources is about $200^{\prime \prime}$. This is about five times the main beam diameter of $36^{\prime \prime}$ at $230 \mathrm{GHz}$. The first side lobes reach from $\approx 30^{\prime \prime}$ to $\approx 90^{\prime \prime}$. Hence, they are covered completely for observations towards the cloud center only. We correct the raw data given in terms of $T_{\mathrm{A}}^{\star}$ (notation of Kutner \& Ulich 1981) by the main beam efficiency, i.e. we approximate the source brightness temperature $T_{\mathrm{B}}$ by the main beam brightness temperature

$T_{\mathrm{B}}(\vartheta) \approx T_{\mathrm{mb}}=\frac{\eta_{\mathrm{f}}}{\eta_{\mathrm{mb}}} T_{\mathrm{A}}^{\star}(\vartheta)$,

$\eta_{\mathrm{f}}$ and $\eta_{\mathrm{mb}}$ being the forward and mainbeam efficiency respectively. The efficiency values are given in Table 2 .

Typical values for the obtained signal-to-noise ratio (SNR) are 30 for $\mathrm{CO}(2-1)$ and 20 for $\mathrm{CO}(3-2)$. Due to bad weather the SNR for the ${ }^{13} \mathrm{CO}(3-2)$ transition is only of the order of 5.

For all objects except CB 231, we localized the position of maximum $\mathrm{CO}$ column density (in the following referred to as the cloud centre), indicated by maximum emission in the optically thin $\mathrm{C}^{18} \mathrm{O}(2-1)$ line. Afterwards, we covered all sources with a course cross of observation points being separated by approximately the width of the main beam and centered on the cloud center. The points of the cross were observed in $\mathrm{CO}(2-1)$ and $\mathrm{CO}(3-2)$. At the cloud center observations were made in the $\mathrm{CO}(2-1), \mathrm{CO}(3-2),{ }^{13} \mathrm{CO}(2-1),{ }^{13} \mathrm{CO}(3-2)$ and $\mathrm{C}^{18} \mathrm{O}(2-1)$ transitions.

For CB 24, CB 25 and CB 202 we were able to take a full map in CO (2-1) (Fig. 1). Due to bad weather conditions, a complete map for CB 231 could not be taken. CB 24 was also mapped in ${ }^{13} \mathrm{CO}(2-1)$.

\section{Data analysis}

We start data analysis with a coarse grid of parameters, centered around values that appear to be reasonable estimates for the observed objects, roughly specified by $10^{3} \mathrm{~cm}^{-3} \leq$ $n_{0} \leq 10^{4} \mathrm{~cm}^{-3}, 9 \mathrm{~K} \leq T \leq 15 \mathrm{~K}, 2 v_{\text {th }} \leq \sigma \leq 3.5 v_{\text {th }}$ and $10^{15} \mathrm{~cm} \leq \ell \leq 10^{18} \mathrm{~cm}$. ( $v_{\text {th }}$ is the mean thermal velocity given by $\left.v_{\text {th }}=\sqrt{2 k T / m}\right)$. For each point of this grid, we calculate the line profiles. These line profiles are then interpolated on a finer grid, dividing each original division into 10 subdivisions. On the fine grid, we apply a standard $\chi^{2}$ method to find the set of parameters, the calculated line profiles of which match the observed ones best. This means that we have to minimize the objective function

$\chi^{2}=\frac{1}{m-n} \sum_{i=1}^{m}\left[\frac{\left\langle I_{v, i}\right\rangle-I_{v, i, \mathrm{obs}}}{\sigma_{i}}\right]^{2}$,

where $\left\langle I_{v, i}\right\rangle$ is the calculated intensity, $I_{v, i, \text { obs }}$ the observed intensity, $\sigma_{i}$ the experimental error, and $m-n$ the number of degrees 

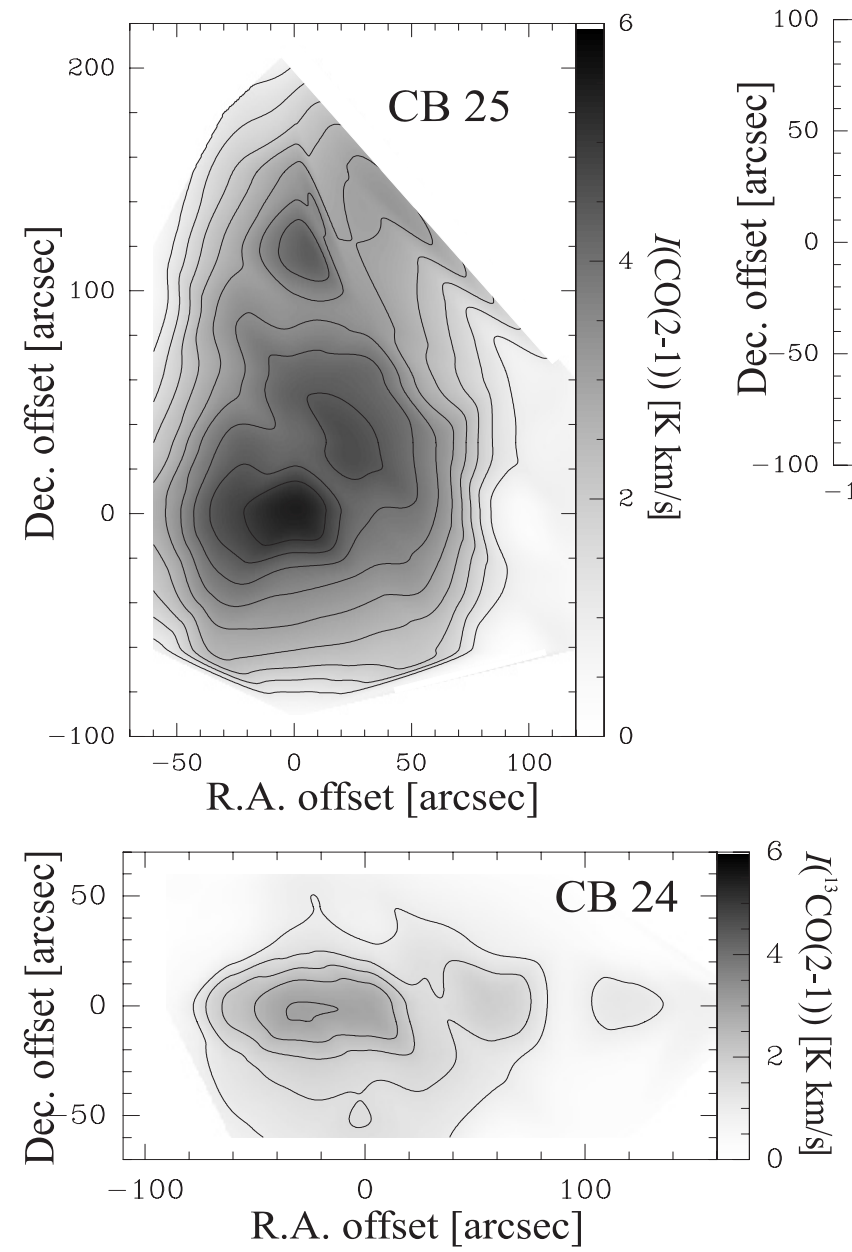
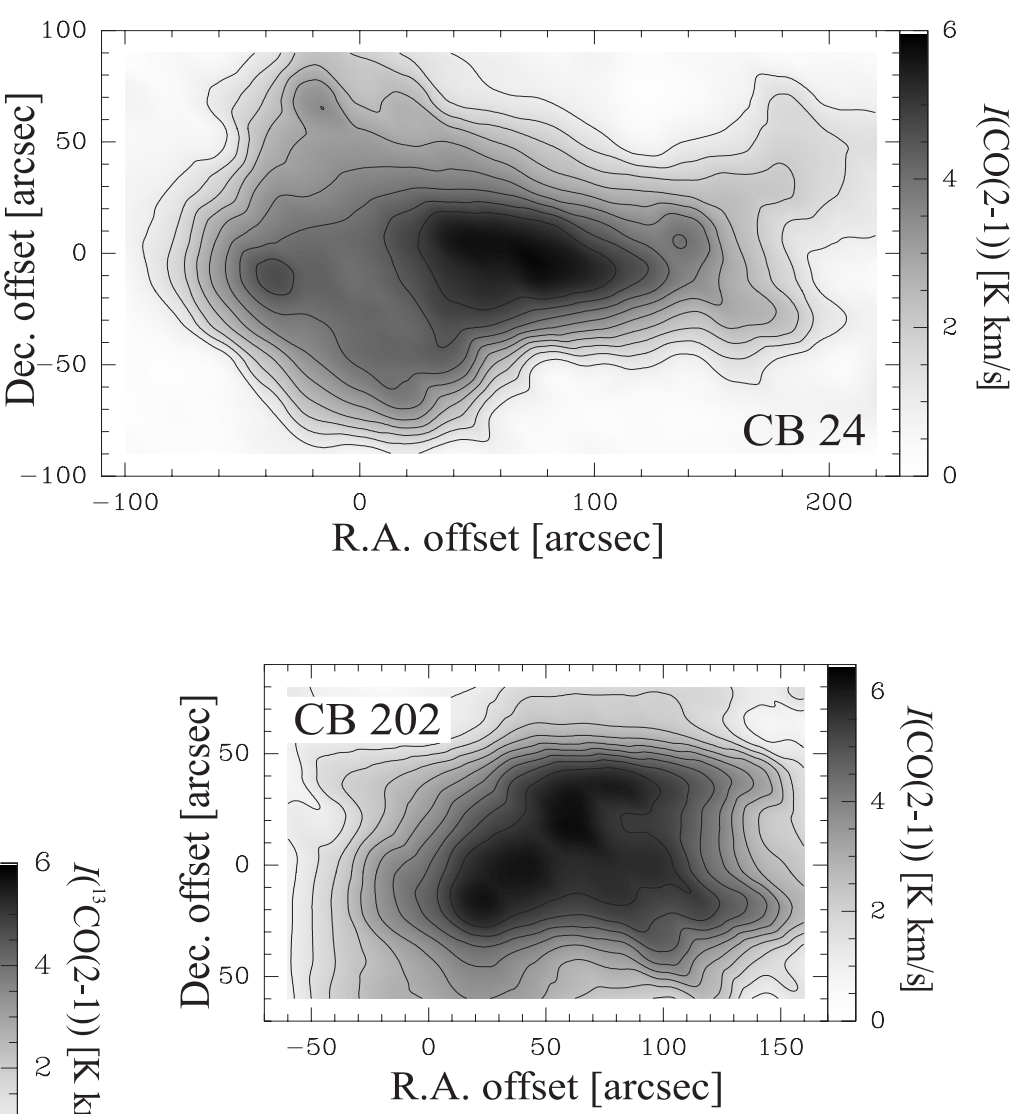

R.A. offset [arcsec]

Fig. 1. Emission from the CO $J=2 \rightarrow 1$ transition for 3 of the 5 Bok-Globules observed. For CB 24 a map in the ${ }^{13} \mathrm{CO}(2-1)$ line is also given. Contours are from $1 \mathrm{~K} \mathrm{~km} \mathrm{~s}^{-1}$ to the maximum value observed in steps of $0.5 \mathrm{~K} \mathrm{~km} \mathrm{~s}^{-1}$.

of freedom ( $m$ is the number of data points and $n$ the number of free parameters, $n=4$ in our case). In the next step, we refine the initial rough grid to a grid enclosing the parameters of this first approximation. We repeat this procedure until an improvement of coincidence can no longer be achieved or we reach parameter ranges for which there exist no stable cloud models. Since our model intrinsically produces symmetric line shapes, we symmetrize the observed profiles for the purpose of comparison with the calculated lines when calculating the $\chi^{2}$ values.

\section{Results}

\subsection{General results}

In this section, our observational data of the individual objects are presented along with the data analysis using the SRTM. At first we used our model to simultaneously fit the observations in the CO (2-1) and CO (3-2) lines for the central line of sight of each cloud. For all objects except CB 213 we were able to find parameters that yield satisfactory coincidence between observational and calculated line profiles. Figure 2 shows the calculated and the observed line profiles for each object. In the upper part of Table 3 we present the values obtained for the central hydrogen density $n_{\mathrm{H}_{2}}(0)$, the cloud temperature $T$, the mean square turbulent velocity in units of the thermal velocity $\sigma / v_{\text {th }}$, the correlation length $\ell$, the cloud mass $M$ and the cloud radius in $\mathrm{CO}$ and $\mathrm{H}_{2}$ respectively.

In the case of $\mathrm{CB} 24$, the $\chi^{2}$ analysis yields two distinct local minima at $T=15 \mathrm{~K}, n_{\mathrm{H}_{2}}(0)=1.8 \times 10^{3} \mathrm{~cm}^{-3}$ and $T=12.4 \mathrm{~K}, n_{\mathrm{H}_{2}}(0)=3.8 \times 10^{3} \mathrm{~cm}^{-3}$, respectively. For CB 25, only a lower limit for the central hydrogen density is given. As the low intensity ratio $T_{\mathrm{B}, \max }(\mathrm{CO}(2-1)) / T_{\mathrm{B}, \max }(\mathrm{CO}(3-2))$ indicates, both lines are optically thick. For a more precise determination of the $\mathrm{H}_{2}$ number density, we have to analyze the lines of the rarer isotopomeres ${ }^{13} \mathrm{CO}$ and $\mathrm{C}^{18} \mathrm{O}$. The need to add the lines of the rarer isotopomeres in our analysis can be also seen in Fig. 3. Here, $\chi^{2}$ is given as a function of the central hydrogen density and the kinetic gas temperature for the globule CB 24. Only the region around the first minimum is shown. The values of $\sigma / v_{\text {th }}$ and $\ell$ are kept constant in this plot. It can be clearly seen that there is a very narrow but extended region in the $T-n_{\mathrm{H}_{2}}$ space for which small values of $\chi^{2}$ are obtained. However, the fit parameters we obtained from the analysis of the lines of the main isotopomer are very similar to the ones that yield a simultaneous fit of all observed rotational transitions. 
Table 3. Upper part: results in the case of simultaneous evaluation of CO (2-1) and CO (3-2). Middle part: corresponding values determined by Turner (1994) using a microturbulent approximation. Lower part: corresponding values derived by a LVG-analysis.

\begin{tabular}{|c|c|c|c|c|c|c|c|}
\hline & $T[\mathrm{~K}]$ & $n_{\mathrm{H}_{2}}(0)\left[10^{3} \mathrm{~cm}^{-3}\right]$ & $\sigma\left[v_{\mathrm{th}}\right]$ & $\ell\left[10^{16} \mathrm{~cm}\right]$ & $M\left[M_{\odot}\right]$ & $R_{\mathrm{H}_{2}}\left[10^{18} \mathrm{~cm}\right]$ & $R_{\mathrm{CO}}\left[10^{18} \mathrm{~cm}\right]$ \\
\hline \multicolumn{8}{|c|}{ SRTM, simultaneous fit of $\mathrm{CO}(2-1)$ and $\mathrm{CO}(3-2)$} \\
\hline CB 24 & $15.4(12.4)$ & $1.8(3.8)$ & $2.25(2.25)$ & $6.8(10)$ & $64(39)$ & $3.3(2.5)$ & $0.2(0.3)$ \\
\hline CB 25 & 10 & $\geq 10$ & 2.5 & 10 & 27 & 2.1 & 0.3 \\
\hline CB 202 & 15 & 2.5 & 2.5 & 5.7 & 71 & 3.4 & 0.3 \\
\hline CB 231 & 10 & 5.0 & 6.4 & 32 & 360 & 7.5 & 1.1 \\
\hline \multicolumn{8}{|c|}{ microturbulent approach (Turner 1994) } \\
\hline CB 24 & 15 & 2.8 & 2.6 & - & $0.4-2.5$ & $0.3-0.6$ & - \\
\hline CB 202 & 15 & 1.6 & 3.0 & - & 20 & 2.0 & - \\
\hline \multicolumn{8}{|l|}{ LVG } \\
\hline CB 24 & 13.3 & 0.8 & - & - & 1.5 & - & 0.58 \\
\hline CB 25 & 9.1 & 40 & - & - & 0.5 & - & 0.11 \\
\hline CB 202 & 10.9 & 1.7 & - & - & 5.0 & - & 0.68 \\
\hline CB 231 & 8.6 & 4.0 & - & - & 31.8 & - & 0.95 \\
\hline
\end{tabular}
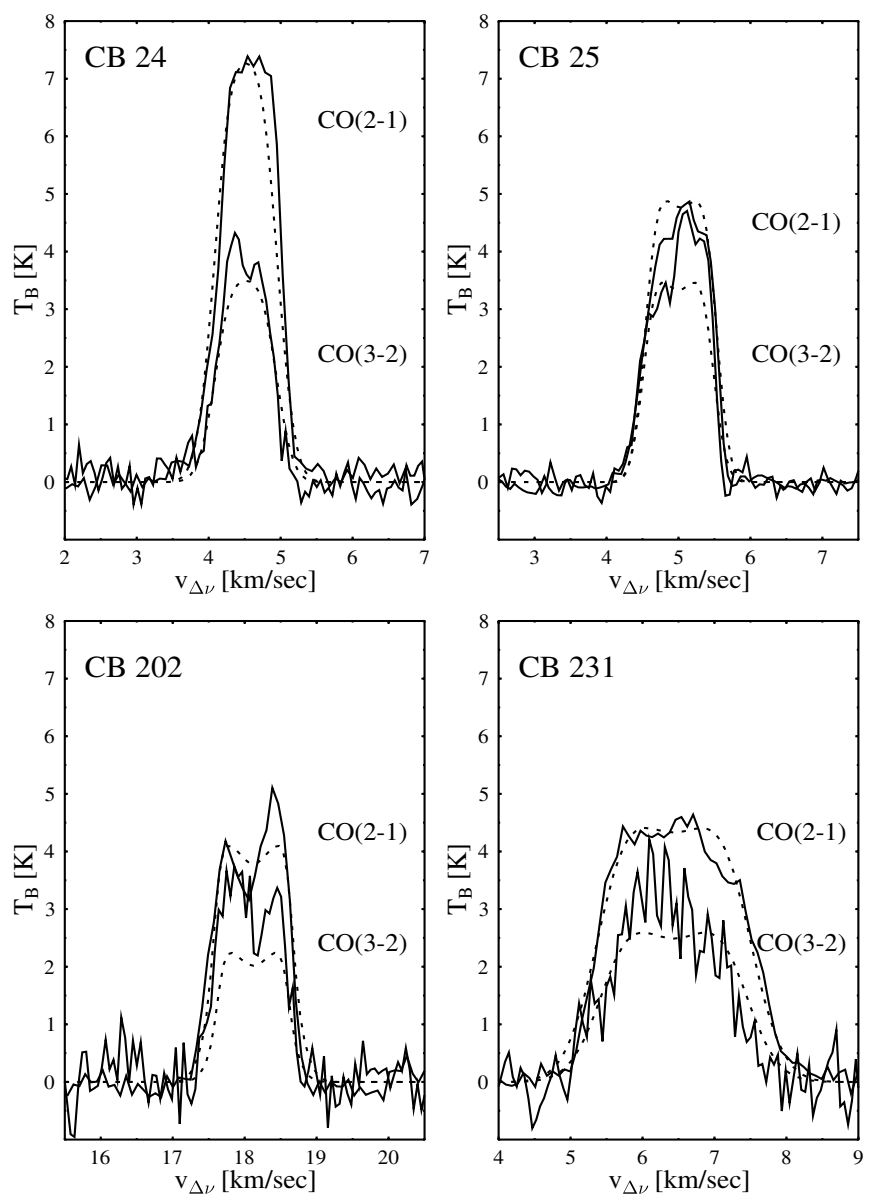

Fig. 2. Observed (solid) and calculated (dotted) $\mathrm{CO} J=3 \rightarrow 2$ and $J=3 \rightarrow 2$ spectra for the central line of sight of each cloud.

For $\mathrm{CB} 24$, the derived radius $R_{\mathrm{CO}}$ is of special interest, since for this globule a reliable value for its distance $D \approx$ $360 \mathrm{pc}$ is known (Peterson \& Clemens 1998). Together with the observed CO radius $R_{\mathrm{obs}} \approx 80^{\prime \prime}$ this distance leads to an

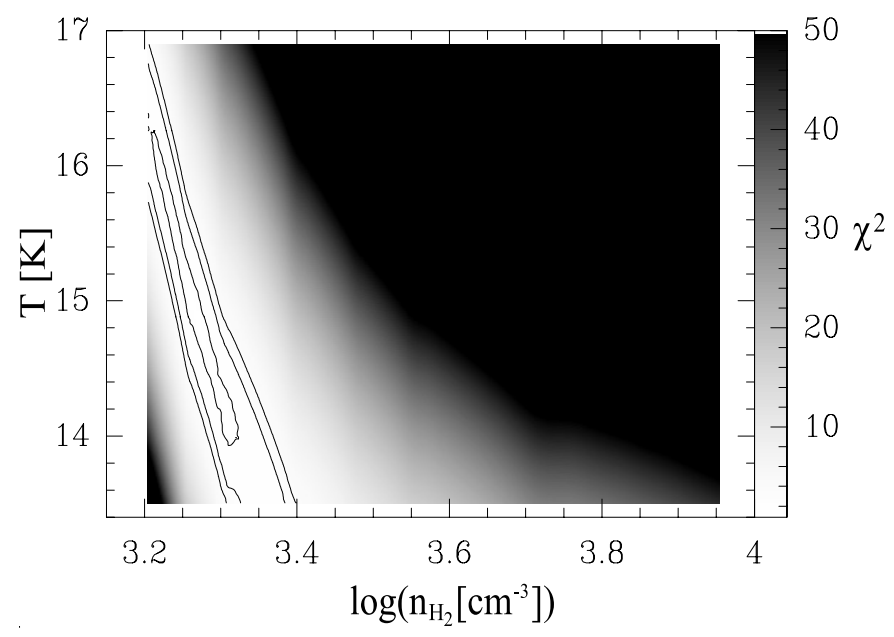

Fig. 3. $\chi^{2}$ for CB 24 as a function of $T$ and $\log n_{\mathrm{H}_{2}}$ for constant $\sigma=$ 2.25 and $\ell=4.64 \times 10^{16} \mathrm{~cm}$. The contours correspond to $\chi^{2}=1,2,3$.

observed cloud radius $R_{\mathrm{obs}} \approx 0.14 \mathrm{pc}$, which is at least of the same order as the theoretical value $R_{\mathrm{CO}} \approx 0.07-0.1 \mathrm{pc}$.

An even lower ratio $T_{\mathrm{B}, \max }(\mathrm{CO}(2-1)) / T_{\mathrm{B}, \max }(\mathrm{CO}(3-2))$ than in the case of CB 25 was found for the globule CB 213, which cannot be explained by our model. The model of an isothermal sphere implies that the density of the gas drops strongly towards the boundary of the cloud. This decrease of the density and the spherical geometry lead to an increased escape probability for the photons at the edge of the cloud, and consequently a gradient in the excitation temperature with cloud depth builds up. This decrease of the excitation temperature towards the cloud's edge becomes more pronounced for the higher rotational transitions, because the higher rotational levels are less effectively populated by collisions of $\mathrm{CO}$ with $\mathrm{H}_{2}$ than the lower ones. As a result, our model does not yield $T_{\mathrm{B}, \max }(\mathrm{CO}(2-1)) / T_{\mathrm{B}, \max }(\mathrm{CO}(3-2))$ intensity ratios near 1.0 as observed for $\mathrm{CB} 213$. In Fig. 4, we give the ratio of the maximum $\mathrm{CO}(2-1)$ and $\mathrm{CO}(3-2)$ line intensities as a function of the central density. For each density, the remaining parameters 


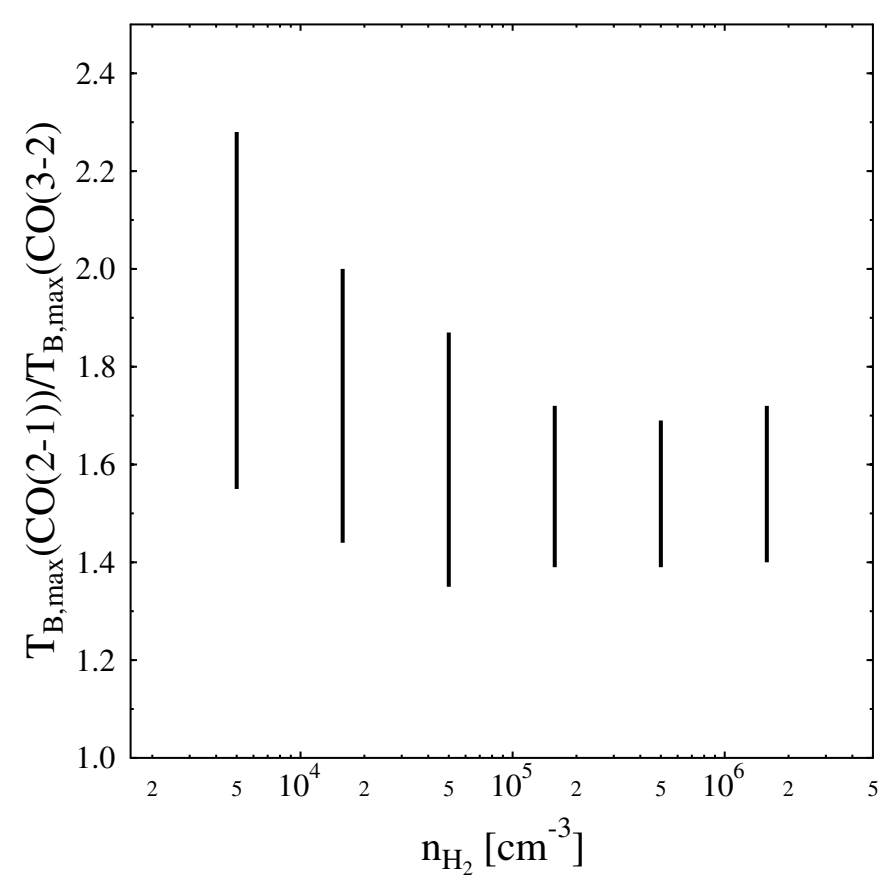

Fig. 4. Ratio of the maximum $\mathrm{CO}(2-1)$ and $\mathrm{CO}(3-2)$ calculated line intensities as a function of the central density. For each density, the remaining parameters have been variied in the limits of the parameter space covered in this analysis.

have been varied in the limits of the parameter space covered in this analysis, leading to $T_{\mathrm{B}, \max }(\mathrm{CO}(2-1)) / T_{\mathrm{B}, \max }(\mathrm{CO}(3-2))$ ratios which do not drop below 1.3. The strong excitation of $\mathrm{CO}(3-2)$ with respect to $\mathrm{CO}(2-1)$ may be taken as an indication of a clumpy structure of the emitting medium rather than a density distribution, which smoothly drops with increasing distance from the cloud center (cf. Hegmann \& Kegel 2000).

\subsection{Correlation effects}

One of the most important objectives of our investigation was to find out to what degree correlation effects are important for the determination of physical parameters. It is not possible to distinguish between the cases of macro-, meso- or microturbulence by only considering the absolute value of the correlation length of the velocity field. One rather has to compare the mean free path of a given photon, $l_{\mathrm{mfp}}$ to the correlation length $\ell$. The microturbulent case may then be characterized by $\ell / l_{\text {mfp }} \ll 1$, the macroturbulent case by $\ell / l_{\mathrm{mfp}} \gg 1$ and the intermediate mesoturbulent case by $\ell / l_{\mathrm{mfp}} \approx 1$. Note that a given velocity field may be macroturbulent for one line and meso- or even microturbulent for another line at the same time, since the mean free path of a photon is essentially the inverse line absorption coefficient. Furthermore, a given velocity field will be classified differently for the line center and the line wings due to the decrease of the absorption coefficient towards the wings.

Under the previous considerations, an appropriate quantity to study the importance of correlation effects is the mean optical depth of a single correlation element at the line center,

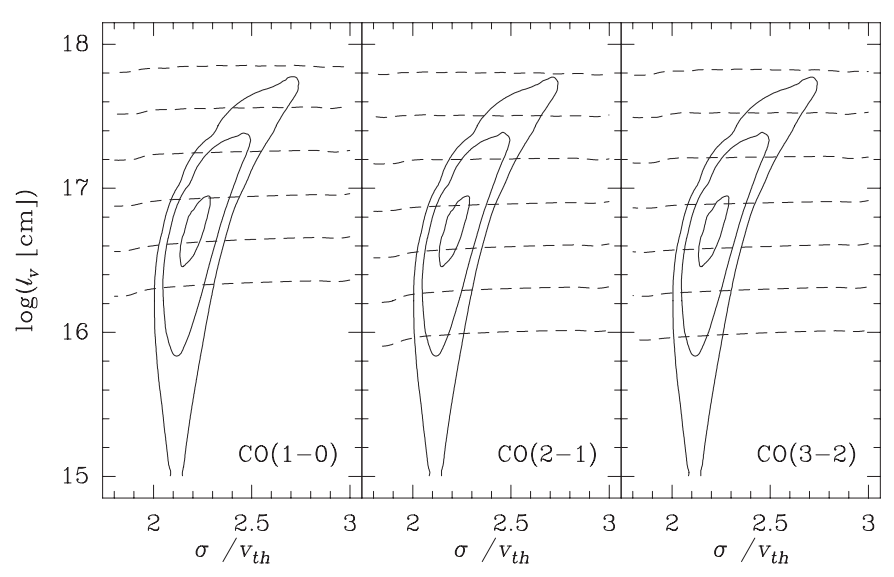

Fig. 5. The ratio $\tau_{\ell}$ of the correlation length of the turbulent velocity field to the mean free path of the photons in the line cnter for CB 24 as a function of $\sigma$ and $\ell . T=15.4 \mathrm{~K}$ and $\log n_{\mathrm{H}_{2}}=1.8 \times 10^{3} \mathrm{~cm}^{-3}$ are kept constant. The dotted contours are $\tau_{\ell}=1,2,4,8,16, \ldots$ The solid lines mark the area, for which good fits are obtained: $\chi^{2}=1,2,3$.

which is the mean absorption coefficient at the line centre times the correlation length:

$\tau_{\ell}=\left\langle\kappa_{v}\right\rangle \ell=\frac{\ell}{\left\langle l_{\mathrm{mfp}}\right\rangle}$.

In terms of this quantity, the above conditions read $\tau_{\ell} \ll 1$ for microturbulence, $\tau_{\ell} \gg 1$ for macroturbulence and $\tau_{\ell} \approx 1$ for mesoturbulence.

We also would like to give an intuitive interpretation of the meaning of the quantity $\tau_{\ell}$ : imagine the line of sight to be divided into several turbulence elements, each of them having a typical scale length $\ell$. In a single representation of the velocity field, the turbulent velocity along the line of sight does not vary significantly within a single turbulence cell. If each turbulence element is optically thin for the photons of a given line, the photons may escape this turbulence element largely unaffected. In this case, the turbulence elements may be exchanged arbitrarily along the line of sight and the two-point-correlation brakes down to the uncorrelated microturbulent limit. If in the opposite limit the correlation length takes values of the order of the cloud size or larger, the whole cloud will be covered by one single turbulence element with one single value for the turbulent velocity $v_{0}$. The emitted line will then be essentially thermally broadened and be centered around $v_{0}$. The mean value of the intensity taken with respect to the velocity will therefore be a convolution of a thermally broadened Gaussian for the individual line with a Gaussian broadened by the mean turbulent velocity for the one-point-probability distribution function. This is what one would expect in the macroturbulent limit.

In Fig. 5, we show a contour plot of $\tau_{\ell}$ in the line and cloud center for different transitions $J+1 \rightarrow J$ in the $\sigma$ - $\ell$-projection of the parameter space (dashed lines). The solid lines enclose the region where a good coincidence between calculations and observations has been found. One should keep in mind that $\tau_{\ell}$ becomes smaller with increasing distance from the cloud center due to the decreasing density towards the cloud's edge. The models that show good coincidence between calculated and observed lines in each case are located in the mesoturbulent 


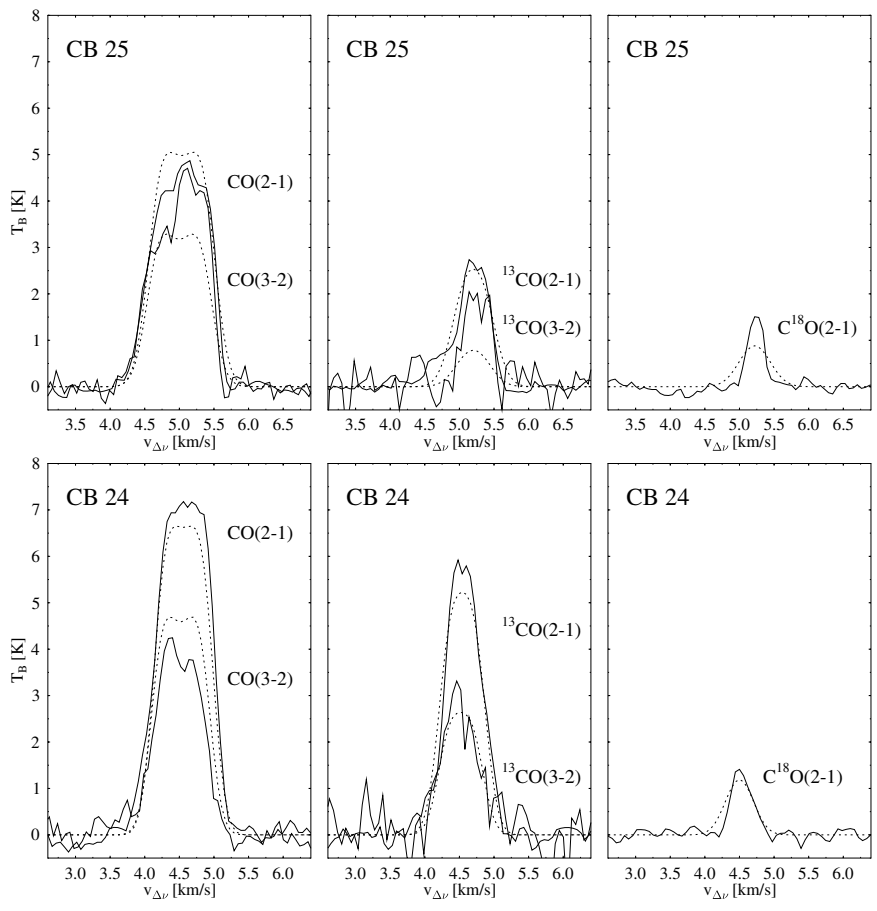

Fig. 6. Observed (solid) and calculated (dotted) $\mathrm{CO},{ }^{13} \mathrm{CO}$, and $\mathrm{C}^{18} \mathrm{O}$ rotational lines for the central line of sight of CB 24 and CB 25.

regime $\tau_{\ell} \approx 1$, i.e. in the regime which is not addressed by microturbulent or LVG approximations.

\subsection{Interpretation of ${ }^{13} \mathrm{CO}$ - and $\mathrm{C}^{18} \mathrm{O}$-lines}

Since the optical depths of the ${ }^{13} \mathrm{CO}$ and $\mathrm{C}^{18} \mathrm{O}$ lines are significantly lower than that of the main isotopomer, it is desirable to include these lines into our analysis. In principle, a simultaneous fit of all lines from the different isotopomers taken at the cloud center allows also to determine the relative abundances, treating them as an additional set of parameters. However, the resulting additional dimensions of parameter space would lead to a tremendous increase of computing time, making the method inefficient for data analysis. For the globules CB 24 and CB 25, a simultaneous fit for all observed lines was performed, using a small set of values for the respective abundances only. For the $\mathrm{CO} /{ }^{13} \mathrm{CO}$ ratio, values between 20 and 89 have been adopted; the $\mathrm{CO} / \mathrm{C}^{18} \mathrm{O}$ ratio was set to a fixed value of $1.7 \times 10^{-7}$.

The analysis of CB 24 yields $T=12.4 \mathrm{~K}$ and $n_{\mathrm{H}_{2}}(0)=7.3 \times$ $10^{3} \mathrm{~cm}^{-3}$. These values are in good agreement with the ones we found for the second minimum of the $\chi^{2}$ analysis of the lines of the main isotopomere in Sect. 6.1. The best fit is obtained for a low $\mathrm{CO} /{ }^{13} \mathrm{CO}$ ratio of 20 . In the case of $\mathrm{CB} 25$, we find $T=10.4 \mathrm{~K}, n_{\mathrm{H}_{2}}(0)=7.3 \times 10^{3} \mathrm{~cm}^{-3}$ and a $\mathrm{CO} /{ }^{13} \mathrm{CO}$ ratio of 89 . The value of the central hydrogen density is somewhat smaller than the lower boundary given in Table 3 .

In the case of $\mathrm{CB} 24$, the model lines match the observed ones rather well, whereas for $\mathrm{CB} 25$ the ${ }^{13} \mathrm{CO}(3-2)$ and the $\mathrm{C}^{18} \mathrm{O}$ (2-1) line could not be fitted satisfactorily (see Fig. 6). The ${ }^{13} \mathrm{CO}(3-2)$ transition appears to be much more excited than in our model calculations, which may be an indication of a clumpy medium (cf. Hegmann \& Kegel 2000). However, the SNR for the ${ }^{13} \mathrm{CO}(3-2)$ transition is very poor. The observed $\mathrm{C}^{18} \mathrm{O}(2-1)$ line appears narrower than the corresponding model line. A possible reason could be that the $\mathrm{C}^{18} \mathrm{O}$ emission originates from a much smaller region than the lines of the more abundant isotopomers. This small region near the dense cloud's center is possibly smaller than the correlation length of the velocity field. For this case, the SRTM no longer can be considered as a good approximation because the results as given by the generalized radiative transfer equation are the expectation values of the intensity. This means that the results of the SRTM are valid only if many turbulence elements are observed at the same time and thus the observed intensity can be considered as an average value.

\subsection{Comparison with other evaluation methods}

When comparing theoretical line profiles with observed ones, the main aim is to estimate the physical conditions within the observed clouds. There are two different types of assumptions entering the calculation of theoretical line profiles. At first, one has to specify the physical cloud model, i.e. the temperature and density stratification, the geometry, the dynamical state, etc. The second type of assumptions refer to the approximations made in solving the radiative transfer problem. The methods currently used to analyze observed line data differ in both aspects and it is not always easy to assess the effects of the different assumptions. In the present analysis, we emphasize the radiative transfer problem while assuming a rather simple cloud model, an isothermal sphere in hydrostatic equilibrium.

In the previous sections we have shown that it is possible to analyze observational data with the SRTM. To what extent do the physical parameters we derive agree with or differ from values derived by other methods? We compare the physical parameters we derived before with the results of two complementary methods: a microturbulent radiative transfer model and the large velocity gradient (LVG) approximation.

\subsubsection{The microturbulent approximation}

The mesoturbulent approximation used in the present investigation differs in two ways from the classical microturbulent approximation. Firstly and most importantly, the SRTM accounts for the effects of a finite correlation length in the stochastic velocity field. Secondly, as follows from Eq. (7), in calculating the absorption line profile $\kappa_{v}(\Delta v)$, complete redistribution is assumed over the thermal profile only, whereas in the classical microturbulent approximation the thermal profile is convolved with the distribution function of the turbulent velocity field and complete redistribution is assumed over the total profile. As a consequence, under NLTE conditions the intensity calculated by means of the generalized radiative transfer equation differs from the microturbulent profile even in the limit $\ell \rightarrow 0$.

To illustrate the effects introduced by the different treatment of the radiative transfer problem, we solved the radiative transfer problem in the classical microturbulent approximation using the same isothermal cloud model as described in Sect. 3. 

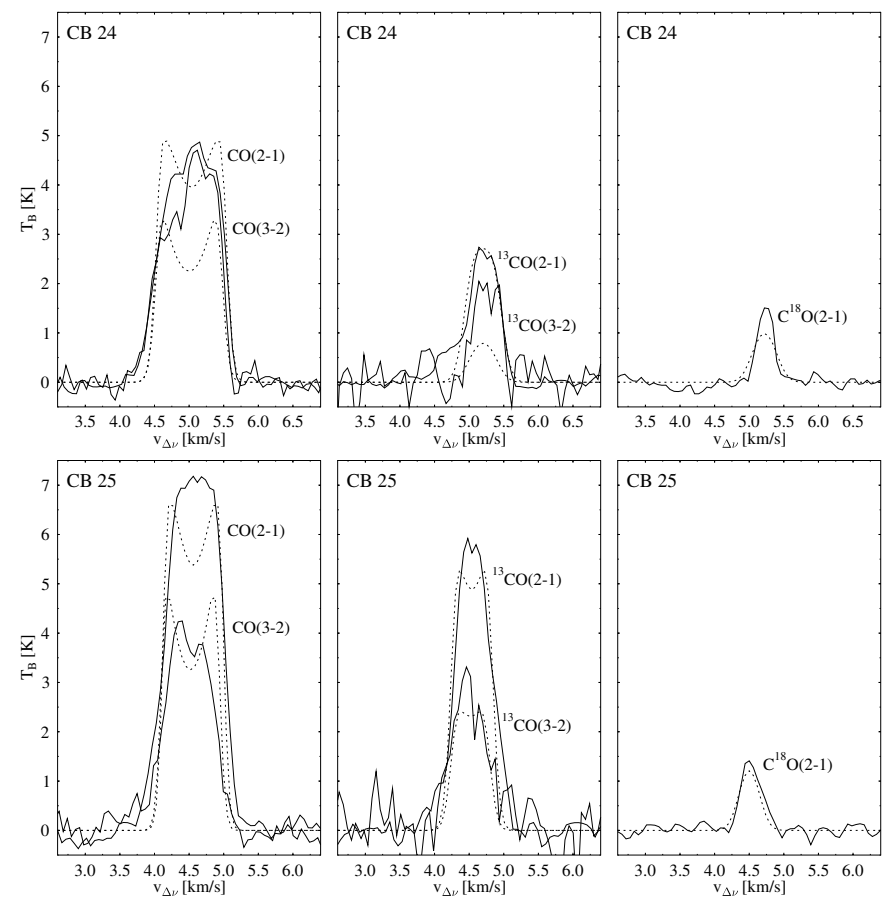

Fig. 7. Observed (solid lines) and calculated (microturbulent model, dotted lines) $\mathrm{CO},{ }^{13} \mathrm{CO}$, and $\mathrm{C}^{18} \mathrm{O}$ rotational lines for the central line of sight of CB 24 and CB 25.

Table 4. Results in the case of a simultaneous evaluation of all observed lines for an isothermal cloud model.

\begin{tabular}{lccccc}
\hline \hline & $T$ & $n_{\mathrm{H}_{2}}(0)$ & $\sigma$ & $N_{\mathrm{CO}}$ & $\chi^{2}$ \\
{$[\mathrm{~K}]$} & {$\left[10^{3} \mathrm{~cm}^{-3}\right]$} & $\begin{array}{c}\sigma \\
{\left[v_{\text {th }}\right]}\end{array}$ & {$\left[10^{17} \mathrm{~cm}^{-2}\right]$} & \\
\hline \multicolumn{5}{l}{ SRTM, simultaneous fit of all observed lines } \\
\hline CB 24 & 12.4 & 7.3 & 2.1 & 3.01 & 2.4 \\
CB 25 & 10.4 & 7.3 & 2.6 & 3.09 & 2.9 \\
\hline microturbulent approach & & & \\
\hline CB 24 & 11.8 & 7.2 & 1.4 & 2.15 & 5.4 \\
CB 25 & 9.8 & 8.4 & 1.9 & 2.49 & 5.6 \\
\hline
\end{tabular}

The results obtained for a simultaneously fit of all observed lines are shown for CB 24 and CB 25 in Fig. 7. The numerical values for the fit parameters are given together with the corresponding SRTM values in Table 4. The results obtained for the central hydrogen density and the gas temperature resemble the SRTM values for both globules analyzed. However, the ratio $\sigma$ of the mean quadratic turbulent velocity to the thermal velocity differs significantly for both models, and as a consequence the $\mathrm{CO}$ column densities are noticeably different.

The lines of the main isotopomere show a strong self absorption in the microturbulent model for both globules which is typical for optically thick lines formed in an inhomogeneous isothermal cloud. Since the excitation temperature drops towards the edge of the cloud (cf. Sect. 6.1), the optically thick central part of the lines shows a lower intensity than those parts of the line which become optically thick deeper within the cloud, where the excitation temperature is higher.
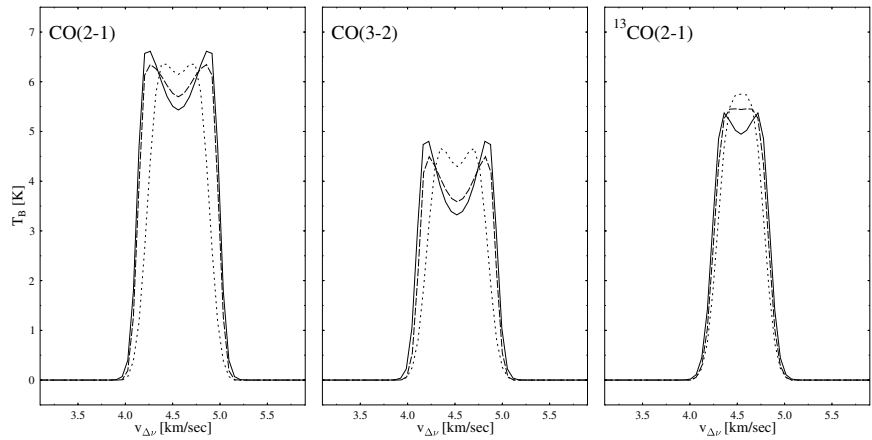

Fig. 8. $\mathrm{CO}$ and ${ }^{13} \mathrm{CO}$ rotational lines for the microturbulent model (solid lines) and the SRTM. The model parameters are the same for both radiative transfer models: $T=11.8 \mathrm{~K}, n_{\mathrm{H}_{2}}(0)=7.4 \times 10^{3} \mathrm{~cm}^{-3}$, and $\sigma / v_{\text {th }}=1.4$. In the case of the SRTM, two different correlation lengths are choosen: $\ell=10^{15} \mathrm{~cm}$ (dashed line) and $\ell=10^{17} \mathrm{~cm}$ (dotted line).

Due to this strong self absorption feature it is difficult to fit the central parts of the observed optically thick lines of CB 24 and $\mathrm{CB} 25$.

To see in which way the differences in the numerical results are caused by the different treatment of the radiative transfer problem, we compare in Fig. 8 the line profiles for the microturbulent approach with the corresponding SRTM profiles adopting two different correlation lengths of the stochastic velocity field. The set of parameters is the same as given in Table 4 for CB 24. Even for the short correlation length (dashed line, $\ell \ll l_{\text {mfp }}$ for the whole line), the SRTM results differ from the profiles obtained in the classical microturbulent approach. There is a diminished intensity in the line wing for the SRTM results. However, the profile for the short correlation length still shows a weak self absorption feature. For the longer correlation length $\ell=1.0 \times 10^{17} \mathrm{~cm}$, the value for which the observations are best reproduced, the lines reveal almost no self absorption. Furthermore, it can be seen that the SRTM profiles become narrower with increasing correlation length. This means that the mean quadratic turbulent velocity derived by fitting the observed lines tend to be much smaller in the microturbulent limit than for a finite correlation length.

\subsubsection{The Turner model}

Physical parameters of CB 24 and CB 202 have also been derived by Turner (1994). We compare our results with those of Turner, although Turner observed different lines $(J=1 \rightarrow 0$ and $J=2 \rightarrow 1$ transitions of $\mathrm{C}^{18} \mathrm{O}$ and $\left.{ }^{13} \mathrm{CO}\right)$. Turner treats the radiative transfer problem in the microturbulent approximation and uses a different physical model. He assumes a hydrostatic model with polytropic index $N=-3$, this means:

$p=K \rho^{2 / 3}$.

To fulfill Eq. (14), the temperature has to increase with decreasing $\rho$ from the centre towards the edge of the cloud (cf. Turner 1992). The assumed increase of the gas temperature towards the edge of the cloud is supported by energy balance arguments. The physical parameters derived by Turner (1994) are given in Table 3 . The central densities and temperatures largely 
agree with our findings. However, due to the different density profiles of the cloud models the estimation of cloud masses and radii differs substantially: for CB 24 the values derived by Turner differ from our values by an order of magnitude, while the differences are smaller in the case of CB 202 (factor of 1.5 for $R$ and 2.5 for $M$ ).

\subsubsection{The LVG approximation}

We use the large velocity gradient approximation in its most commonly used form, assuming a cloud in homologous collapse (Goldreich \& Kwan 1974). Under this assumption, the solution of the NLTE-radiative transfer problem can be easily solved with an escape probability method, since it yields the same isotropic escape probability for all points of the cloud. The line shapes of the calculated lines match the observations less well than the SRTM lines. The physical parameters derived from the LVG-analysis also differ substantially from the SRTM-values (Table 3).

This comparison between the SRTM, the microturbulent and the LVG approximations shows that the choice of the radiative transfer model has an influence on the derived physical parameters like hydrogen density, gas temperature or column density. Hence, the uncertainty for the derived parameters does not only depend on the quality of the observational data or the statistical errors resulting from the respective fitting procedures. It also depends to a high degree on the assumptions of the underlying radiative transfer model.

\section{Summary}

The present paper has two main objectives: to investigate whether and to what extent the stochastic radiative transfer model (SRTM) as described in Paper III is suitable for the analysis of observational millimeter- and submillimeter-line data and to what degree the derived physical parameters agree with results from standard evaluation methods, such as microturbulent approaches or the LVG-analysis.

Observations were carried out at the Heinrich-HertzTelescope in Arizona, taking line data in the rotational transitions of $\mathrm{CO}(J=2 \rightarrow 1, J=3 \rightarrow 2),{ }^{13} \mathrm{CO}(J=2 \rightarrow 1$, $J=3 \rightarrow 2)$, and $\mathrm{C}^{18} \mathrm{O}(J=2 \rightarrow 1)$ for five spherical starless Bok-Globules, CB 24, CB 25, CB 202, CB 213, and CB 231.

We are able to fit the observed $\mathrm{CO}(2-1)$ and $\mathrm{CO}(3-2)$ line profiles for all objects except CB 213 with the SRTM and to derive physical parameters for the observed objects. In two cases (CB 24 and CB 25) we carried out a simultaneous analysis of all observed lines.

Particular observations, such as an over excited $\mathrm{CO}(3-2)$ transition (CB 213) or over excited ${ }^{13} \mathrm{CO}$ (3-2) (CB 25) may be taken as an indication that the emitting medium has a clumpy structure. For an inhomogeneous medium with a high density contrast, the radiation of the higher rotational lines is emitted from dense clumps, where the high rotational levels can be populated by collisions of $\mathrm{CO}$ with $\mathrm{H}_{2}$. As has been shown by Hegmann \& Kegel (2000), this can lead to a noticeable overall increase of the CO (3-2)/CO (2-1) line ratio. Taking into account a clumpy cloud structure would further reduce the self-absorption, as has been shown by Juvela (1997). Therefore, we find it promising to reanalyse the observational data with a model taking into account density fluctuations rather than velocity fluctuations.

We compared the physical parameters derived with the SRTM to the findings of a microturbulent approximation, and to the results given by a standard LVG evaluation method. The values we obtain for the central density and the cloud temperature are in rather good agreement with microturbulent results, while we find significant differences in the derived CO column densities. However, the LVG-model yields noticeably different values for all physical parameters. In particular, the densities derived by means of the LVG-code tend to be systematically lower than the SRTM values.

In addition, we compared our results with the the findings of Turner (1994), who uses a different cloud model to analyze ${ }^{13} \mathrm{CO}$ and $\mathrm{C}^{18}$ rotational lines of CB 24 and CB 202. Taking only the lines of the main isotopomere into account, the SRTM values for the central density and the cloud temperature are in good agreement with the results of Turner. However, we find significant differences in the derived cloud masses and radii. Surprisingly, the differences in the central density and gas temperature for the two cloud models become more significant if the lines of the rarer isotopomers ${ }^{13} \mathrm{CO}$ and $\mathrm{C}^{18} \mathrm{O}$ are taken into account.

These results point out that the physical parameters derived from observational data always depend strongly on the assumptions behind the model used for data analysis. These systematic uncertainties may be much more important than uncertainties due to statistical errors or noisy data. This is especially true for parameters like the overall mass or the radius which depend very sensitively on the cloud model. The degree of sophistication of the model used in analyzing the data should therefore match quality of the observational data.

\section{Outlook}

We consider the following research projects in context with the SRTM to be promising for future work:

- According to the observational evidence, a reanalysis of the observational data should be carried out, also taking into account correlated density fluctuations as described by Hegmann \& Kegel (2000).

- Another promising objective would be to extend the underlying cloud model, considering e.g. a more realistic temperature profile.

- To extend the SRTM to tackle cases where only a few turbulence elements contribute to the observed line. Levshakow et al. $(1997,1999)$ have shown that this problem can be solved by an inverse Monte Carlo method, maintaining the statistical properties of the velocity field. It appears to be a challenging task to extend their formalism to also include NLTE effects self-consistently.

- The generalized radiative transfer equation used in the SRTM considers the velocity component along the line of sight only. However, in the case of NLTE, the intensity is also correlated to the velocity components perpendicular to 
the line of sight. In the actual form of the SRTM, we use an approximation introduced in Paper I. For a detailed review of this approximation, see also Gail et al. (1975). In order to test the impact of the assumptions made in the SRTM on the calculated line profiles, a 3-dimensional Gaussian representation of the velocity field can be used, and the resulting line profiles can be compared to actual SRTM profiles. Since all marginal distributions of a multidimensional Gaussian distribution are again Gaussian, the velocity field along any given line of sight would be described by Eqs. (1) and (2) in this model, too (cf. Hengel 2002).

Acknowledgements. This work was supported in part by the German Bundesministerium für Bildung, Wissenschaft, Forschung und Technologie (BMBF) in project No 053FM13A and by the DFG in the Sonderforschungsbereich 555 (Teilprojekt B8). We thank our referee M. Juvela for a thoughtful and helpful critique of this work.

\section{References}

Albrecht, M. A., \& Kegel, W. H. 1987, A\&A, 176, 317 (Paper I) Blitz, L. 1987, in Physical Processes in Interstellar Clouds, ed. G. M. Morfill, \& M. Scholer (Reidel Verlag)
Gail, H.-P., Hundt, E., Kegel, W. H., Schmid-Burgk, J., \& Traving, G. 1974, A\&A, 32, 65

Gail, H.-P., Sedlmayr, E., \& Traving, G. 1975, A\&A, 44, 421

Genzel, R. 1992, in The Galactic Interstellar Medium, ed. W. B. Burton, B. G. Elmegreen, \& R. Genzel (Springer Verlag)

Goldreich, P., \& Kwan, J. 1974, ApJ, 189, 441

Hegmann, M., \& Kegel, W. H. 2000, A\&A, 359, 405

Hengel, C. 2002, Ph.D. Thesis, Frankfurt a.M. University

Juvela, M. 1997, A\&A, 322, 943

Kegel, W. H., Piehler, G., \& Albrecht, M. A. 1993, A\&A, 270, 407 (Paper II)

Kutner, M. L., \& Ulich, B. L. 1981, ApJ, 250, 341

Leung, C.-M., \& Liszt, H. S. 1976, ApJ, 208, 732

Levshakov, S. A., Kegel, W. H., \& Mazets I.E. 1997, MNRAS, 287, 802

Levshakov, S. A., Kegel, W. H., \& Takahara, F. 1999, MNRAS, 302, 707

Peterson, D. E., \& Clemens, D. P. 1998, AJ, 116, 881

Piehler, G., \& Kegel, W. H. 1995, A\&A, 297, 841 (Paper III)

Sobolev, V. V. 1960, Moving envelopes of stars (Cambridge: Harvard University Press)

Turner, B. E. 1994, ApJ, 420, 661

Turner, B. E., Lanping, Xu, \& Rickard, L. J. 1992, ApJ, 391, 158

van Dishoeck, E. F., \& Black, J. H. 1988, ApJ, 334, 771 